\title{
Éthique de la recherche : l'examen doit se faire une seule fois et bien se faire
}

$\mathrm{L}$ es conseils d'éthique de la recherche jouent un rôle essentiel dans la protection des droits et du mieux-être des participants aux recherches. Chercheurs, instituts de recherche et bailleurs de fonds se plaignent toutefois que le système ne fonctionne pas, et surtout que les essais multicentriques sont surexaminés et embourbés dans la bureaucratie parce que chaque centre effectue ses propres examens indépendants.

Un simple calcul indique que pour un essai portant sur 20 centres, toutes les parties qui participent à l'examen de l'éthique pourront devoir y consacrer au total de 300 à 500 heures-personnes. Étant donné ce coût d'opportunité important, on espérerait trouver des preuves claires indiquant qu'un exercice qui consomme tellement de maind'œuvre protégerait plus efficacement les patients. Or, ces données n'existent pas. La contribution d'un nombre important de comités peut en fait avoir un effet indésirable sur les participants à la recherche, par exemple, en rendant les formulaires de consentement plus complexes ${ }^{1}$. De plus, la nécessité de procéder à de multiples examens indépendants réduit le temps et le savoir-faire que l'on pourrait consacrer à l'examen et à la surveillance d'autres études. On retire aussi du temps au soin des patients, à la recherche et à d'autres activités utiles des soins de santé, sans avantage démontré.

En Amérique du Nord, le processus d'examen de l'éthique dépend en général d'examens locaux que l'on juge essentiels pour garantir que les décisions conformes à l'éthique reflètent les conditions et la culture locales. Or, même si les conditions locales sont importantes dans des essais multicentriques, les questions de culture locale le sont moins. De plus, en raison des contraintes reliées aux coûts et aux ressources en soins de santé, on demande de plus en plus aux comités de l'éthique de la recherche «Disposons-nous localement des ressources et du savoir-faire nécessaires pour entreprendre cette étude?» plutôt que «Cette étude est-elle respectueuse de l'éthique?» Il y a encore plus important : les grandes études nationales et internationales soulèvent souvent des problèmes importants de responsabilité civile, d'imputabilité et de responsabilité, ainsi que des préoccupations liées à la surveillance une fois l'étude lancée. Même si les conseils locaux d'éthique de la recherche peuvent donner un avis sur ces questions, il arrive souvent que certains d'entre eux ne soient pas entendus, faute d'orientation nationale ou de capacité de s'exprimer collectivement.

Nous devons repenser le système d'examen de l'éthique et transformer un processus basé sur des comités locaux autonomes en une collaboration interdépendante de comités locaux œuvrant dans le contexte d'un seul système national. Même si une multitude de règles et de règlements variables régissent l'éthique de la recherche en Amérique du Nord, tous permettent la collaboration entre les conseils d'éthique, ce qui permettrait de créer un seul mécanisme d'examen décentralisé. Le nouveau système national remplacerait les examens multiples totalement indépendants et offrirait aux comités locaux l'appui d'un modèle d'examen ouvert interactif. Dans une telle refonte, nous ne préconisons pas que les établissements locaux cèdent le droit d'approuver les études. Nous envisageons plutôt un processus simplifié et pertinent à l'échelle locale une fois l'examen complet de l'éthique effectué au niveau central. L'examen de l'éthique de la recherche devrait désormais avoir pour mantra de «se faire une seule fois et bien se faire».

Afin d'entreprendre ces changements, il faudrait accorder d'abord la priorité à l'harmonisation des normes institutionnelles d'un bout à l'autre du Canada. On pourrait créer des formulaires communs afin d'alléger le fardeau administratif imposé par les essais multicentriques. Une fois les normes acceptées en place, les établissements et les comités de l'éthique pourraient collaborer afin de revoir des études et de mettre en commun leurs constatations. L'adoption de points de repère clairs afin de garantir la pertinence et la qualité des examens favoriserait en outre l'imputabilité. La mise sur pied d'un programme national d'éducation à l'intention des nouveaux membres de comités de l'éthique de la recherche et des chercheurs en santé, ainsi que l'éducation permanente à l'intention des membres plus chevronnés, pourraient faciliter ces changements et aider à simplifier et à améliorer le rôle de tous les conseils d'éthique de la recherche. L'affichage de toutes les décisions sur des sites web publics favoriserait la transparence, importante pour les établissements qui visent à protéger le public et à garder sa confiance.

Il y a eu des progrès à l'échelle régionale au Canada. Québec et Terre-Neuve-et-Labrador ont adopté des systèmes d'examen centralisé de l'éthique. Le comité ontarien d'éthique de la recherche sur le cancer (affilié à l'Ontario Institute for Cancer Research) revoit les essais sur le cancer dans toute la province. Pour qu'il y ait des améliorations dans tout le système, nous croyons toutefois qu'une solution nationale est à la fois opportune et nécessaire.

Au Canada, il ne se produira rien de tout cela sans le leadership d'institutions nationales comme les Instituts de recherche en santé du Canada, le Groupe en éthique de la recherche et d'autres conseils scientifiques. Il faudra aussi que les milieux scientifiques et de l'éthique fassent preuve de leadership pour bâtir la confiance et susciter la volonté de collaborer. Il faudrait rechercher des solutions semblables aux ÉtatsUnis. Si l'Europe au complet envisage de collaborer en matière d'examen de l'éthique, il n'y a aucune raison pour que nous ne puissions faire de même au Canada, voire dans toute l'Amérique du Nord.

\section{Paul Hébert MD MHSc}

Rédacteur en chef

$J A M C$

\section{Raphael Saginur MD}

Président, Comité de la gouvernance

Comité ontarien d'éthique de la recherche sur le cancer

(affilié à l'Ontario Institute for Cancer Research)

Avec l'équipe de rédaction de l'éditorial

(Matthew B. Stanbrook MD PhD, Ken Flegel MDCM MSc, Noni MacDonald MD MSc et Amir Attaran LLB DPhil)

Intérêts concurrents : Voir www.cmaj.ca/misc/edboard.shtml pour les déclarations de l'équipe de rédaction de l'éditorial. Paul Hébert est employé des Instituts de recherche en santé du Canada. Les opinions exprimées dans cet article sont celles des auteurs et non celles des Instituts de recherche en santé du Canada. Raphael Saginur est cochercheur au Canadian Network for the Governance of Ethical Health Research Involving Humans, financé par les Instituts de recherche en santé du Canada.

Traduit par le Service de traduction de l'AMC.

\section{RÉFÉRENCE}

1. Burman W, Breese P, Weis S, et al.; Tuberculosis Trials Consortium. The effects of local review on informed consent documents from a multicenter clinical trials consortium. Control Clin Trials 2003;24:245-55. 